\title{
IMP-3 expression in melanocytic lesions
}

\begin{abstract}
Background: Insulin-like growth factor-II mRNA-binding protein 3 (IMP-3), a member of the insulin-like growth factor mRNA-binding protein family, is expressed in several human malignancies, including melanomas. However, the expression of IMP-3 has not been explored in melanoma in situ, various histologic subtypes of invasive melanomas and atypical Spitz tumors.
\end{abstract}

Methods: IMP-3 immunostain was performed in 157 melanocytic lesions.

Results: Nearly all benign (8/8), dysplastic (8/8) and Spitz nevi (8/9) were negative for IMP-3. Focal IMP-3 positivity was observed in 5/12 melanoma in situ and 4/15 superficial melanomas (Breslow depth $\leq 1 \mathrm{~mm})$. Half $(10 / 20)$ of deep melanomas (Breslow depth $>1 \mathrm{~mm}$ ) and 25/52 metastatic melanomas demonstrated strong IMP-3 staining. IMP-3 expression differs significantly between non-desmoplastic melanomas (superficial and deep) and benign or dysplastic or Spitz nevi ( $p=0.0427$, respectively). Four of 23 desmoplastic melanomas expressed IMP-3, which was significantly different from deep melanomas $(p=0.0109)$. IMP-3 stained 7 of 10 atypical Spitz tumors. The difference between atypical Spitz tumors and Spitz nevi was statistically significant $(\mathrm{p}=0.0256)$.

Conclusion: A malignant circumstance, such as non-desmoplastic melanoma or atypical Spitz tumor, can be inferred when IMP-3 is expressed, suggesting potential diagnostic value of IMP-3 in melanocytic lesions.

Yu L, Xu H, Wasco MJ, Bourne PA, Ma L. IMP-3 expression in melanocytic lesions.

J Cutan Pathol 2010; 37: 316-322. (C) 2009 John Wiley \& Sons A/S.

\section{Limin $\mathbf{Y u}^{1}$, Haodong $\mathbf{X} \mathbf{u}^{3}$, Matthew J. Wasco ${ }^{1}$, Patricia A. Bourne $^{3}$ and Linglei $\mathrm{Ma}^{1,2}$}

\author{
${ }^{1}$ Department of Pathology, and \\ ${ }^{2}$ Department of Dermatology, University of \\ Michigan Medical Center, Ann Arbor, MI, \\ USA \\ ${ }^{3}$ Department of Pathology, University of \\ Rochester Medical Center, Rochester, \\ NY, USA
}

Linglei Ma, Department of Pathology, Dermatopathology Division, University of Michigan, M3260, Medical Sciences 1, 1301 Catherine Street, Ann Arbor, MI 48109-0602, USA

Tel: +17347644460

Fax: +17347644690

e-mail: lingleim@umich.edu

Accepted for publication August 12, 2009
Malignant melanoma is a life-threatening cutaneous cancer. Its incidence and mortality has risen worldwide. ${ }^{1}$ The diagnosis of melanocytic lesions is mainly based on a number of well-defined histology criteria, but remains one of the most challenging areas in dermatopathology. Interobserver differences are not infrequently encountered for difficult lesions. ${ }^{2}$ Despite advances in melanoma research, no reliable diagnostic and/or prognostic markers have yet been identified.

Insulin-like growth factor-II (IGF-II) mRNAbinding protein 3 (IMP-3), also known as $\mathrm{K}$ homology domain-containing protein overexpressed in cancer (KOG) or L523S, is a member of the insulin-like growth factor mRNA-binding protein family. ${ }^{3}$ Located on chromosome 7p11.5, IMP-3 gene encodes a 580 -amino acid protein that binds to IGF-II transcripts and is involved in the posttranscriptional regulation of cell proliferation during embryogenesis. Its expression is limited in normal mature tissue. ${ }^{3,4}$ Since IMP-3 was first cloned from pancreatic carcinomas, it has been detected in cervical adenocarcinoma, renal cell carcinoma, urothelial carcinoma and other malignancies. ${ }^{5-7}$ Additionally, IMP-3 was shown to be an independent prognostic marker in renal cell carcinoma and its expression in early stage lesions could help identify patients with high potential to develop metastasis. ${ }^{5}$ 
Data from in vitro IMP-3 knockdown study and from IMP-3 administration in patients with lung cancer indicate that IMP-3 may be a promising therapeutic target for human malignancies. ${ }^{8,9} \mathrm{~A}$ recent study demonstrated that IMP-3 was expressed in malignant melanomas but not in nevi. ${ }^{10}$ However, the expression of IMP-3 in melanoma in situ (MIS), desmoplastic melanoma and atypical Spitz tumor (AST) has not been examined in the literature. Interestingly, IMP-1, another member of IMP family protein, was recently found to be elevated in primary human melanomas and melanoma cell lines. ${ }^{11}$

In this study, we investigated the expression of IMP-3 in a spectrum of cutaneous melanocytic lesions, including benign/dysplastic/Spitz nevus, MIS, primary melanoma (non-desmoplastic and desmoplastic melanoma), AST and metastatic melanoma. We further assessed the diagnostic and prognostic utility of IMP-3 in melanocytic lesions.

\section{Materials and methods}

Case selection

This study was approved by the Institutional Review Board at the University of Michigan Health System. A broad range of melanocytic lesions was identified through a search of the pathology database from the Department of Pathology at the University of Michigan. As shown in Table 1, the study group included 8 benign nevi, 8 dysplastic nevi, 9 Spitz nevi, 12 MIS, 58 primary melanomas (35 non-desmoplastic and 23 desmoplastic melanomas), 52 metastatic melanomas and 10 ASTs. The histologic subtypes of non-desmoplastic melanomas were superficial spreading (21), lentigo maligna (5), nodular (4), nevoid (1), Spitzoid (1) and acral lentiginous types (3). Based on the Breslow depth, non-desmoplastic melanomas were further separated into superficial (depth $\leq 1.0 \mathrm{~mm}$ ) and deep melanomas (depth $>1.0 \mathrm{~mm}$ ). Sentinel lymph node (SLN) biopsies were performed on 19 of 20 patients with deep melanomas. Five showed nodal metastases, while one patient with negative SLNs had bone metastasis (Table 4). All desmoplastic melanomas had a Breslow depth greater than $1.0 \mathrm{~mm}$. SLN biopsies were performed on 21 cases, 3 of which were positive. All ASTs had a depth greater than $1 \mathrm{~mm}$. Among eight cases with SLN biopsies available, six showed microscopic tumor deposits (minute foci), one had $<5 \%$ surface area involved and one was negative.

The hematoxylin and eosin-stained slides were reviewed to confirm the diagnoses. Important prognostic information, such as Breslow depth, mitotic rate and SLN status, was collected for primary invasive melanomas (see Table 4). The pathologic and clinical stages were determined using the American Joint Committee on Cancer (AJCG) staging system. ${ }^{12}$ Unstained whole tissue sections were available for all nevi, all MIS, most non-desmoplastic melanomas, five desmoplastic melanomas and six metastatic melanomas. Three non-desmoplastic melanomas and the rest of desmoplastic and metastatic melanomas were derived from tissue microarray sections.

\section{Immunohistochemistry}

Formalin-fixed paraffin-embedded $4-\mu \mathrm{m}$ tissue sections were deparaffinized and pretreated with $3 \% \quad \mathrm{H}_{2} \mathrm{O}_{2}$ and Tris buffer saline (TBS). After antigen retrieval, the sections were incubated with a monoclonal mouse anti- IMP-3/L523S antibody (1:100 dilution; clone 69.1; Dako, Carpinteria, CA, USA) at room temperature for 40-60 min and then with EnVision+ System horseradish peroxidase (HRP) labeled polymer conjugated to goat antimouse (Dako) for $30 \mathrm{~min}$. Staining was achieved with 3-amino-9-ethylcarbazole (AEC)+SubstrateChromogen (Dako) for $10 \mathrm{~min}$. Tissue from large cell neuroendocrine carcinoma of the lung was used as positive control.

Table 1. Clinical characteristics of the patients

\begin{tabular}{lcll}
\hline & $\mathrm{N}$ & Average age (range) & $\mathrm{M}: \mathrm{F}(\%)$ \\
\hline Benign nevus & 8 & $34(14-46)$ & $25 / 75$ \\
Dysplastic nevus & 8 & $39(20-71)$ & $50 / 50$ \\
Spitz nevus & 9 & $20(5-61)$ & $56 / 44$ \\
Melanoma in situ & 12 & $67(22-85)$ & $58 / 42$ \\
Non-desmoplastic melanoma & 35 & $57(29-94)$ & $54 / 46$ \\
Desmoplastic melanoma & 23 & $62(23-92)$ & $70 / 30$ \\
Metastatic melanoma & 52 & $54(20-83)$ & $67 / 33$ \\
Atypical Spitz tumor & 10 & $15(6-41)$ & $30 / 70$ \\
\hline
\end{tabular}

$\mathrm{N}$, total numbers of cases; $\mathrm{M}$, male; $\mathrm{F}$, female. 


\section{Yu et al.}

Table 2. The percentage of tumor cells stained with IMP-3 in melanocytic lesions

\begin{tabular}{lrrllll}
\hline & $\mathrm{N}$ & Negative & $<10 \%$ & $10-50 \%$ & $>50 \%$ & $\geq 10 \%$ \\
\hline Nevus & 25 & $24(96.0 \%)$ & $1(4.0 \%)$ & $0(0 \%)$ & $0(0 \%)$ & $0(0 \%)^{*}$ \\
$\quad$ benign nevus & 8 & $8(100 \%)$ & $0(0 \%)$ & $0(0 \%)$ & $0(0 \%)$ & $0(0 \%)$ \\
$\quad$ dysplastic nevus & 8 & $8(100 \%)$ & $0(0 \%)$ & $0(0 \%)$ & $0(0 \%)$ & $0(0 \%)$ \\
$\quad$ Spitz nevus & 9 & $8(88.9 \%)$ & $1(11.1 \%)$ & $0(0 \%)$ & $0(0 \%)$ & $0(0 \%)$ \\
Melanoma in situ & 12 & $7(58.3 \%)$ & $5(41.7 \%)$ & $0(0 \%)$ & $0(0 \%)$ & $0(0 \%)$ \\
Non-desmoplastic melanoma & 35 & $21(60.0 \%)$ & $1(2.9 \%)$ & $7(20.0 \%)$ & $6(17.1 \%)$ & $13(37.1 \%)$ \\
$\quad$ melanoma $\leq 1 \mathrm{~mm}$ & 15 & $11(73.3 \%)$ & $1(6.7 \%)$ & $3(20.0 \%)$ & $0(0 \%)$ & $3(20.0 \%)$ \\
$\quad$ melanoma $>1 \mathrm{~mm}$ & 20 & $10(50.0 \%)$ & $0(0 \%)$ & $4(20.0 \%)$ & $6(30.0 \%)$ & $10(50.0 \%)$ \\
Desmoplastic melanoma & 23 & $19(82.6 \%)$ & $1(4.3 \%)$ & $2(8.7 \%)$ & $1(4.3 \%)$ & $3(13.0 \%)^{\dagger}$ \\
Metastatic melanoma & 52 & $25(48.1 \%)$ & $2(3.8 \%)$ & $2(3.8 \%)$ & $23(44.2 \%)$ & $25(48.1 \%)$ \\
Atypical Spitz tumor & 10 & $3(30.0 \%)$ & $2(20.0 \%)$ & $2(20.0 \%)$ & $3(30.0 \%)$ & $5(50 \%)^{\ddagger}$ \\
\hline
\end{tabular}

${ }^{*} p=0.0251$ when compared with non-desmoplastic melanoma.

${ }^{\dagger} p=0.0109$ when compared with melanoma $>1 \mathrm{~mm}$.

$\ddagger p=0.0256$ when compared with Spitz nevus.

Cytoplasmic staining was considered positive for IMP-3. The percentage of positive cells was recorded as negative $(0),<10 \%(1+), 10-50 \%(2+)$ or $>50 \%$ $(3+)$. The staining intensity was graded as weak $(1+)$, moderate $(2+)$ or strong $(3+)$. Two pathologists (LY and $\mathrm{LM}$ ) independently recorded the staining results. The two had complete concordance in scoring a case positive or negative and reached general consensus as regard to the percentage and intensity of positive staining.

\section{Statistical analysis}

Statistical analysis was carried out using SAS 8.2 software (SAS Institute Inc., Cary, NG). The Fisher's exact test was used to assess the differences in IMP-3 expression among various melanocytic lesions. A p value $<0.05$ was considered statistically significant.

\section{Results}

In normal skin, IMP-3 did not label any epidermal or dermal structures (data not shown).

The clinical characteristics of the 157 melanocytic lesions are listed in Table 1 . Table 2 and 3 summarize IMP-3 immunostain results. One Spitz nevus displayed IMP-3 positivity in less than $10 \%$ of the lesional cells. All other benign, dysplastic and Spitz nevi were negative for IMP-3 (Fig. 1A-C).
Five of $12(41.7 \%)$ cases of MIS demonstrated moderate IMP-3 staining in less than $10 \%$ of the tumor cells. The positive cells were arranged as isolated single cells or rare small aggregates (Fig. 2A). Similarly, focal IMP-3 positivity was detected in a subset (4/15) of superficial melanomas (depth $\leq 1 \mathrm{~mm}$ ) with most cases (3/4) demonstrating strong intensity in at least $10 \%$ of the tumor cells (Fig. 2B). Compared to MIS, the in situ component of superficial melanomas above the dermal invasion was marked as continuous linear arrays rather than discrete single cells (Fig. 2B). Half (10/20) of deep melanomas (depth $>1.0 \mathrm{~mm}$ ) expressed IMP-3 in at least $10 \%$ of the tumor cells with most $(8 / 10)$ having moderate to strong intensity (Fig. 2C). The expression of IMP-3 in non-desmoplastic melanomas (superficial and deep) was significantly different from that in benign nevi, dysplastic nevi or Spitz nevi $(p=0.0427$ individually; $p=0.0251$ when all nevi combined). The difference between superficial and deep melanomas was not significant $(\mathrm{p}=0.0916)$. As shown in Table 4, the expression of IMP-3 in non-desmoplastic melanomas appeared to be independent of age, sex, histologic subtype and other important prognostic factors. Interestingly, only a small subset of desmoplastic melanomas (4/23; $17.4 \%$ ) expressed IMP-3 with moderate to strong intensity (Fig. 2D). The staining was significantly different between desmoplastic and non-desmoplastic deep melanomas $(\mathrm{p}=0.0109)$.

Fig. 1. (A) A benign nevus, (B) a dysplastic nevus and $(\mathrm{C})$ a Spitz nevus stained negative for IMP-3 $(\times 400)$.
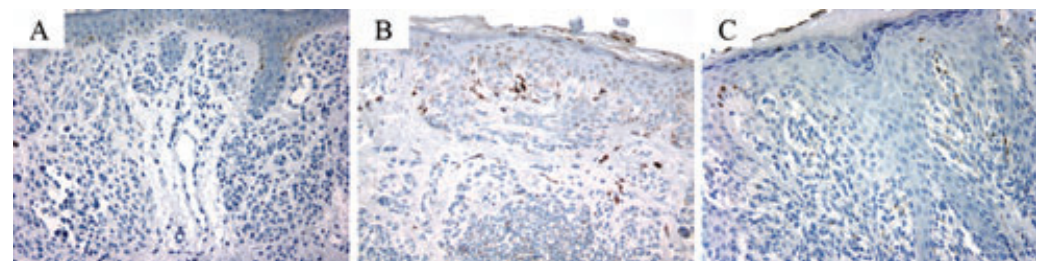

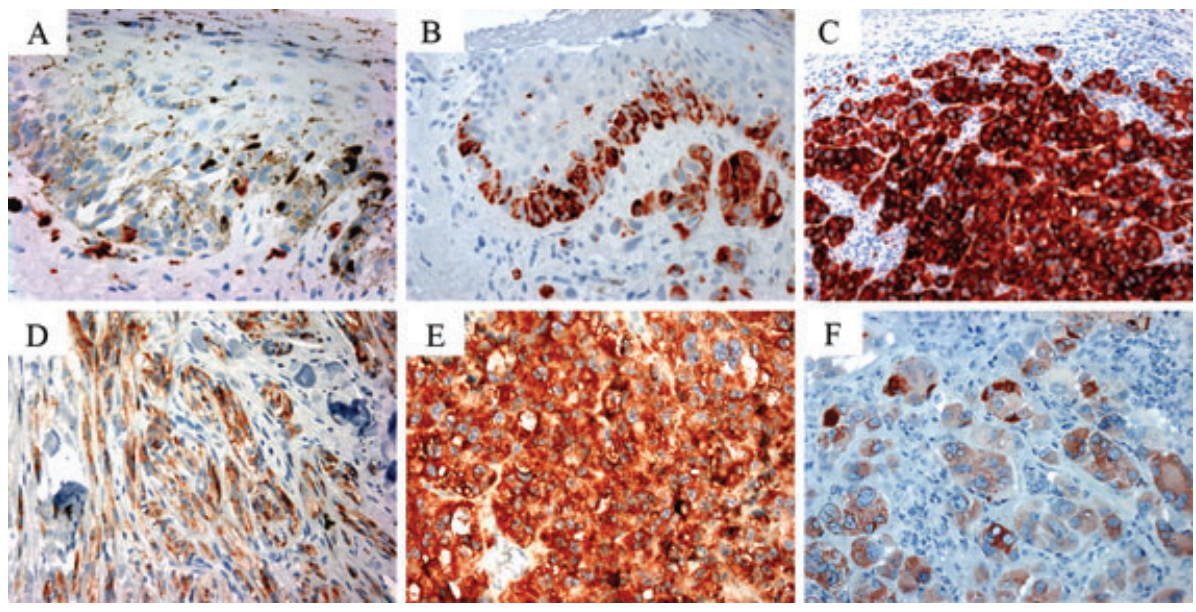

Fig. 2. (A) A case of MIS demonstrated weak IMP-3 positivity with single cell pattern. (B) Focal and strong IMP-3 staining in superficial melanoma (Breslow depth $\leq 1 \mathrm{~mm}$ ). The in situ component of superficial melanomas was stained mostly as confluent tumor nests. (C) Deep melanoma (Breslow depth $>1.0 \mathrm{~mm}$ ) showing diffuse and strong IMP-3 staining. (D) A case of desmoplastic melanoma with moderate IMP-3 positivity. (E) Strong IMP-3 staining in metastatic melanoma. (F) Moderate IMP-3 labeling in a case of AST $(\times 400)$.

Table 3. IMP-3 staining intensity in melanocytic lesions

\begin{tabular}{|c|c|c|c|c|c|}
\hline & $\mathrm{N}$ & Negative & Weak & Moderate & Strong \\
\hline Nevus & 25 & $24(96.0 \%)$ & $0(0 \%)$ & $1(4.0 \%)$ & $0(0 \%)$ \\
\hline benign nevus & 8 & $8(100 \%)$ & $0(0 \%)$ & $0(0 \%)$ & $0(0 \%)$ \\
\hline dysplastic nevus & 8 & $8(100 \%)$ & $0(0 \%)$ & $0(0 \%)$ & $0(0 \%)$ \\
\hline Spitz nevus & 9 & $8(88.9 \%)$ & $0(0 \%)$ & $1(11.1 \%)$ & $0(0 \%)$ \\
\hline Melanoma in situ & 12 & $7(58.3 \%)$ & $0(0 \%)$ & $5(41.7 \%)$ & $0(0 \%)$ \\
\hline Non-desmoplastic melanoma & 35 & $21(60.0 \%)$ & $3(8.6 \%)$ & $2(5.7 \%)$ & $9(25.7 \%)$ \\
\hline melanoma $\leq 1 \mathrm{~mm}$ & 15 & $11(73.3 \%)$ & $1(6.7 \%)$ & $0(0 \%)$ & $3(20.0 \%)$ \\
\hline melanoma >1 mm & 20 & $10(50.0 \%)$ & $2(10.0 \%)$ & $2(10.0 \%)$ & $6(30.0 \%)$ \\
\hline Desmoplastic melanoma & 23 & $19(82.6 \%)$ & $0(0 \%)$ & $3(13.0 \%)$ & $1(4.4 \%)$ \\
\hline Metastatic melanoma & 52 & $25(48.1 \%)$ & $1(1.9 \%)$ & $8(15.4 \%)$ & $18(34.6 \%)$ \\
\hline Atypical Spitz tumor & 10 & $3(30.0 \%)$ & $3(30.0 \%)$ & $3(30.0 \%)$ & $1(10.0 \%)$ \\
\hline
\end{tabular}

Similar to non-desmoplastic deep melanomas, 27/52 (51.9\%) metastatic melanomas were positive for IMP-3 (Fig. 2E). Nearly all (26/27) showed moderate to strong staining and most $(23 / 27)$ stained diffusely. No significant difference was attained between deep melanomas and metastatic melanomas.

Unlike Spitz nevus, most ASTs (7/10; 70\%) expressed IMP-3, with five showing staining in at least $10 \%$ of tumor cells and six having weak to moderate intensity (Fig. 2F). There was a statistical difference between AST and Spitz nevus $(p=0.0256)$. But IMP-3 expression between AST and deep melanoma was not significantly different $(\mathrm{p}=0.686)$.

\section{Discussion}

Our study investigated for the first time the expression of IMP-3 in MIS, desmoplastic melanoma and AST.

The expression of IMP-3 was significantly different between primary non-desmoplastic melanomas and benign/dysplastic/Spitz nevi $(\mathrm{p}=0.0427$ individually; $\mathrm{p}=0.0251$ when all nevi combined). Our findings confirm Pryor's observation ${ }^{10}$ and suggest that a diagnosis of melanomas as opposed to melanocytic nevi may be inferred when IMP-3 is strongly expressed. Interestingly, Pryor et al. ${ }^{10}$ observed that 2 of 10 Spitz nevi showed diffuse IMP-3 staining, while only 1 Spitz nevus in our study showed positivity in $<10 \%$ of tumor cells. It is unclear what may have attributed to this difference. Future studies with more Spitz nevi included would be helpful to address the issue.

We observed discrete and isolated single IMP-3positive cells in MIS. However, because the staining was limited to $<10 \%$ of tumor cells, IMP-3 is less likely to be of any use in separating MIS from dysplastic nevus. Conventional histology remains the most important tool in diagnosing MIS.

Recently, IMP-3 was shown to be an independent prognostic marker in renal cell carcinoma and urothelial carcinoma to identify patients who had

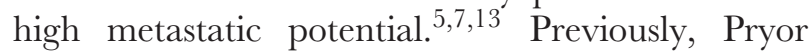




\section{Yu et al.}

Table 4. Clinical characteristics and IMP-3 staining in primary non-desmoplastic melanomas

\begin{tabular}{|c|c|c|c|c|c|c|c|c|c|c|c|c|}
\hline Case & Age & Gender & Type & TNM stage & $\begin{array}{l}\text { Breslow } \\
\text { depth } \\
(\mathrm{mm})\end{array}$ & Ulceration & Regression & $\begin{array}{l}\text { Mitotic } \\
\text { rate/mm² }\end{array}$ & TIL & SLN & $\begin{array}{l}\text { \% IMP-3 } \\
\text { staining }\end{array}$ & $\begin{array}{l}\text { IMP-3 } \\
\text { intensity }\end{array}$ \\
\hline 1 & 74 & M & SSM & T1aNxMx & 0.26 & no & no & insuf & 2 & ND & $2+$ & $3+$ \\
\hline 2 & 55 & M & SSM & T1aNxMx & 0.27 & no & yes & insuf & 2 & ND & 0 & 0 \\
\hline 3 & 81 & $F$ & SSM & T1aNxMx & 0.34 & no & yes & 0 & 2 & ND & $2+$ & $3+$ \\
\hline 4 & 52 & M & SSM & T1aNxMx & 0.35 & no & no & 0 & 1 & ND & 0 & 0 \\
\hline 5 & 73 & M & LMM & T1aNxMx & 0.41 & no & no & 4 & 1 & ND & $2+$ & $3+$ \\
\hline 6 & 70 & M & LMM & T1aNxMx & 0.43 & no & no & 0 & 1 & ND & 0 & 0 \\
\hline 7 & 41 & $\mathrm{~F}$ & SSM & T1aNxMx & 0.44 & no & no & insuf & 2 & ND & 0 & 0 \\
\hline 8 & 45 & $\mathrm{~F}$ & SSM & T1aNxMx & 0.48 & no & no & 0 & 2 & ND & 0 & 0 \\
\hline 9 & 63 & $\mathrm{~F}$ & SSM & T1aNxMx & 0.55 & no & yes & insuf & 1 & ND & 0 & 0 \\
\hline 10 & 59 & M & SSM & T1bNxMx & 0.55 & no & yes & insuf & 2 & ND & 0 & 0 \\
\hline 11 & 61 & $\mathrm{~F}$ & SSM & T1aNxMx & 0.56 & no & yes & 0 & 2 & ND & 0 & 0 \\
\hline 12 & 40 & $M$ & SSM & T1aNxMx & 0.60 & no & no & insuf & 1 & ND & 0 & 0 \\
\hline 13 & 48 & $M$ & SSM & T1aNxMx & 0.63 & no & yes & 0 & 2 & ND & 0 & 0 \\
\hline 14 & 57 & M & SSM & T1aNxMx & 0.64 & no & yes & insuf & 1 & ND & 0 & 0 \\
\hline 15 & 53 & M & LMM & T1bNxMx & 0.82 & no & no & 0 & 2 & ND & $1+$ & $1+$ \\
\hline 16 & 74 & $\mathrm{~F}$ & SSM & T2aNOMx & 1.01 & no & no & 1 & 2 & neg & $2+$ & $2+$ \\
\hline 17 & 43 & $F$ & ALM & T2bN2bM1a & 1.15 & yes & no & 0 & 1 & pos & 0 & 0 \\
\hline 18 & 73 & $M$ & SSM & T2aNOMx & 1.16 & no & no & 0 & 1 & neg & 0 & 0 \\
\hline 19 & 66 & $M$ & SSM & T2aNOMx & 1.16 & no & no & 0 & 1 & neg & $3+$ & $3+$ \\
\hline 20 & 64 & $M$ & SSM & T2aNOMx & 1.46 & no & no & 0 & 1 & neg & $2+$ & $2+$ \\
\hline 21 & 85 & M & LMM & T2aNOMx & 1.57 & no & no & 3 & 2 & neg & $3+$ & $3+$ \\
\hline 22 & 51 & $\mathrm{~F}$ & SSM & T3aN0M1c & 1.7 & no & no & 2.5 & 2 & neg* & 0 & 0 \\
\hline 23 & 76 & M & NO & T3aNOMx & 2.4 & no & no & 6 & 1 & neg & 0 & 0 \\
\hline 24 & 44 & $M$ & SSM & T3aNOMx & 2.4 & no & yes & 0 & 1 & neg & 0 & 0 \\
\hline 25 & 94 & $\mathrm{~F}$ & LMM & T3aNxMx & 2.5 & no & no & 3 & 1 & ND & $3+$ & $3+$ \\
\hline 26 & 39 & $\mathrm{~F}$ & SSM & T3aN2bMx & 2.5 & no & no & 2 & 1 & pos & $3+$ & $3+$ \\
\hline 27 & 72 & $M$ & NO & T3aN2bMx & 2.9 & no & yes & 11 & 2 & pos & $3+$ & $3+$ \\
\hline 28 & 40 & $\mathrm{~F}$ & $\mathrm{NE}$ & T3aN3Mx & 3.05 & no & no & 3.5 & 1 & pos & 0 & 0 \\
\hline 29 & 51 & $\mathrm{~F}$ & SSM & T3aNOMx & 3.6 & no & no & 2 & 1 & neg & $2+$ & $1+$ \\
\hline 30 & 29 & $M$ & NO & T3aNOMx & 3.65 & no & no & 1.5 & 1 & neg & 0 & 0 \\
\hline 31 & 50 & $\mathrm{~F}$ & SSM & T3aNOMx & 3.75 & no & no & 1.5 & 1 & neg & $3+$ & $3+$ \\
\hline 32 & 51 & $\mathrm{~F}$ & ALM & T4bNOMx & 4.1 & yes & no & 3.5 & 1 & neg & 0 & 0 \\
\hline 33 & 34 & $\mathrm{~F}$ & NO & T4aNOMx & 4.5 & no & no & 1 & 2 & neg & 0 & 0 \\
\hline 34 & 38 & $\mathrm{~F}$ & SPM & T4aN1bMx & 5.25 & no & no & 1 & 2 & pos & $2+$ & $1+$ \\
\hline 35 & 46 & $M$ & ALM & T4bNOMx & 7.1 & yes & no & 3 & 1 & neg & 0 & 0 \\
\hline
\end{tabular}

*Bone metastasis identified.

SSM, superficial spreading melanoma; LMM, lentigo maligna melanoma; ALM, acral lentiginous melanoma; NE, nevoid melanoma; N0, nodular melanoma; SPM, Spitzoid melanoma; TNM, Pathologic T (tumor) stage, N (nodal), M (metastasis); insuf, insufficient tumor volume for count; TIL, tumor infiltrating lymphocytes (1, non-brisk; 2, brisk); ND, not done.

et al. ${ }^{10}$ found that IMP-3 was expressed at lower levels in superficial melanomas than deep melanomas, but the difference between the two was not statistically significant. In concordance with their findings, we also noted stronger and more diffuse IMP-3 staining in deep melanomas than in superficial melanomas. However, there was no statistical difference between superficial and deep melanomas $(p=0.0916)$. In addition, when deep melanomas were compared at incremental Breslow depth (Table 4), no significant correlation was detected between IMP-3 levels and tumor depths, other melanoma prognostic factors or clinical stages.
Our findings suggest that IMP-3 is probably of no value in predicting melanomas with greater risk to metastasize and/or shorter survival intervals. IMP-3 does not seem to play a role in melanoma tumor progression. Future studies with additional cases are necessary to substantiate our observation.

Desmoplastic melanoma is an uncommon fibrosing variant of melanoma, which usually reacts with S100 protein, but is often negative for other melanocytic markers. ${ }^{14}$ It differs from conventional melanoma in its higher local recurrence rate and relatively infrequent involvement of regional lymph node. However, the survival rates for desmoplastic 
melanoma patients are similar to those for patients with other types of melanomas at comparable thickness. ${ }^{15-17}$ It was recently shown that $\gamma-\mathrm{H} 2 \mathrm{AX}$, a marker of activated DNA damage, was overexpressed in most primary cutaneous melanomas except desmoplastic melanomas. ${ }^{18}$ Our observation of lower IMP-3 expression in desmoplastic melanomas compared to other deep melanomas $(p=0.0109)$ is interesting. We suspect that the unique desmoplastic stromal response in desmoplastic melanoma may be related to the limited expression of many common molecules that are upregulated in other melanomas.

AST is a borderline melanocytic lesion with uncertain biologic potential. ${ }^{19}$ It shows overlapping histologic features seen in both Spitz nevus and Spitzoid melanoma. AST generally does not meet the diagnostic threshold for melanoma, but demonstrates concerning features, such as large size, asymmetry, aberrant dermal growth pattern, incomplete maturation, cytologic atypia, increased dermal mitotic rates and deep dermal mitoses. ${ }^{19-21}$ Unlike conventional melanoma, patients with AST carry a favorable prognosis, despite a high incidence of microscopic SLN deposits. ${ }^{22}$ Occasionally, differentiating AST from melanoma or Spitz nevus can be problematic. Among a number of tests (i.e. p53, Ki-67 index and fatty acid synthase) studied in the past, comparative genomic hybridization $(\mathrm{CGH})$ appears to hold promise in determining the malignant potential of some controversial melanocytic lesions, including AST. ${ }^{23-26}$ In this study, we found that the expression of IMP-3 was significantly different between Spitz nevus and AST $(\mathrm{p}=0.0256)$, indicating IMP-3 may be helpful in differentiating AST from Spitz nevus. Although deep melanoma showed stronger IMP-3 staining intensity than AST, the lack of statistical difference argues against its diagnostic value in differentiating the two.

In summary, this is the first study to demonstrate the expression of IMP-3 in MIS, desmoplastic melanoma and AST. Our findings confirm the potential diagnostic utility of IMP-3 in differentiating melanocytic nevus from melanoma and in separating Spitz nevus from AST, when a positive IMP-3 expression is observed. As IMP-3 was expressed at high levels in deep melanoma and metastatic melanoma, it may serve as an intriguing therapeutic target for malignant melanomas.

\section{References}

1. Ries LMD, Krapcho M, Stinchcomb DG, et al. SEER cancer statistics review, 1975-2005. Bethesda, MD: National Cancer Institute, 2005.
2. Corona R, Mele A, Amini M, et al. Interobserver variability on the histopathologic diagnosis of cutaneous melanoma and other pigmented skin lesions. J Clin Oncol 1996; 14: 1218.

3. Nielsen FG, Nielsen J, Christiansen J. A family of IGF-II mRNA binding proteins (IMP) involved in RNA trafficking. Scand J Clin Lab Invest Suppl 2001; 234: 93.

4. Mueller-Pillasch F, Pohl B, Wilda M, et al. Expression of the highly conserved RNA binding protein KOC in embryogenesis. Mech Dev 1999; 88: 95.

5. Jiang Z, Chu PG, Woda BA, et al. Analysis of RNA-binding protein IMP3 to predict metastasis and prognosis of renalcell carcinoma: a retrospective study. Lancet Oncol 2006; 7: 556.

6. Li C, Rock KL, Woda BA, Jiang Z, Fraire AE, Dresser K. IMP3 is a novel biomarker for adenocarcinoma in situ of the uterine cervix: an immunohistochemical study in comparison with pl6(INK4a) expression. Mod Pathol 2007; 20: 242.

7. Sitnikova L, Mendese G, Liu Q et al. IMP3 predicts aggressive superficial urothelial carcinoma of the bladder. Clin Cancer Res 2008; 14: 1701 .

8. Liao B, Hu Y, Herrick DJ, Brewer G. The RNA-binding protein IMP-3 is a translational activator of insulin-like growth factor II leader-3 mRNA during proliferation of human K562 leukemia cells. J Biol Chem 2005; 280: 18517.

9. Wang T, Fan L, Watanabe Y, et al. L523S, an RNA-binding protein as a potential therapeutic target for lung cancer. Br J Cancer 2003; 88: 887.

10. Pryor JG, Bourne PA, Yang Q Spaulding BO, Scott GA, $\mathrm{Xu} \mathrm{H}$. IMP-3 is a novel progression marker in malignant melanoma. Mod Pathol 2008; 21: 431.

11. Thomas AJ, Erickson CA. The making of a melanocyte: the specification of melanoblasts from the neural crest. Pigment Cell Melanoma Res 2008; 21: 598.

12. Greene FLPD, Fleming ID, Fritz A, Balch CM, Haller DG, Morrow M, eds. AJCG cancer staging manual, 6th ed. New York: Springer, 2002.

13. Jiang Z, Lohse CM, Chu PG, et al. Oncofetal protein IMP3: a novel molecular marker that predicts metastasis of papillary and chromophobe renal cell carcinomas. Cancer 2008; 112: 2676.

14. Busam KJ. Cutaneous desmoplastic melanoma. Adv Anat Pathol 2005; 12: 92.

15. Quinn MJ, Crotty KA, Thompson JF, Coates AS, O'Brien CJ, McCarthy WH. Desmoplastic and desmoplastic neurotropic melanoma: experience with 280 patients. Cancer 1998; 83: 1128.

16. Smithers BM, McLeod GR, Little JH. Desmoplastic, neural transforming and neurotropic melanoma: a review of 45 cases. Aust N Z J Surg 1990; 60: 967.

17. Jain S, Allen PW. Desmoplastic malignant melanoma and its variants. A study of 45 cases. Am J Surg Pathol 1989; 13: 358.

18. Wasco MJ, Pu RT, Yu L, Su L, Ma L. Expression of gammaH2AX in melanocytic lesions. Hum Pathol 2008; 39: 1614.

19. Barnhill RL. The Spitzoid lesion: rethinking Spitz tumors, atypical variants, 'Spitzoid melanoma' and risk assessment. Mod Pathol 2006; 19: S21.

20. Barnhill RL, Argenyi ZB, From L, et al. Atypical Spitz nevi/tumors: lack of consensus for diagnosis, discrimination from melanoma, and prediction of outcome. Hum Pathol 1999; 30: 513.

21. Farmer ER, Gonin R, Hanna MP. Discordance in the histopathologic diagnosis of melanoma and melanocytic nevi between expert pathologists. Hum Pathol 1996; 27: 528. 


\section{Yu et al.}

22. Ludgate MW, Fullen DR, Lee J, et al. The atypical Spitz tumor of uncertain biologic potential: a series of 67 patients from a single institution. Cancer 2009; 115: 631.

23. Kapur P, Selim MA, Roy LC, Yegappan M, Weinberg AG, Hoang MP. Spitz nevi and atypical Spitz nevi/tumors: a histologic and immunohistochemical analysis. Mod Pathol 2005; 18: 197.

24. Kanter-Lewensohn L, Hedblad MA, Wejde J, Larsson O. Immunohistochemical markers for distinguishing Spitz nevi from malignant melanomas. Mod Pathol 1997; 10: 917.
25. Bastian BC, LeBoit PE, Hamm H, Brocker EB, Pinkel D. Chromosomal gains and losses in primary cutaneous melanomas detected by comparative genomic hybridization. Cancer Res 1998; 58: 2170.

26. Harvell JD, Kohler S, Zhu S, Hernandez-Boussard T, Pollack JR, van de Rijn M. High-resolution array-based comparative genomic hybridization for distinguishing paraffinembedded Spitz nevi and melanomas. Diagn Mol Pathol 2004; 13: 22. 